\title{
Gabriel Gustav Valentin, Pionier der Berner Physiologie
}

\author{
Von Paul Müller
}

Als Gabriel Gustav Valentin am 16. Juni 1836 26jährig zum Lehrstuhlinhaber für Physiologie und Zootomie ernannt wurde, waren auf den Tag anderthalb Jahre seit der feierlichen Eröffnung der Universität Bern am 15. November 1834 verstrichen ${ }^{2}$.

Nach der Pariser Juli-Revolution von 1830 hatte die auch in Bern um sich greifende Unrast das aristokratische Ancien Régime entfernt und einen - an angelsächsischen Vorbildern sich orientierenden - demokratischen Staat mit gemäßigten Volksrechten entstehen lassen. Die auf den Beginn der Restaurationszeit zurückreichende, vom Patriziat 1805 gegründete Akademie mit ihren typischen Standesprivilegien wurde durch die liberale Universität abgelöst ${ }^{1}$.

Die Aufteilung des damals üblichen einheitlichen Lehrstuhls für Anatomie in zwei personell selbständige Lehrbereiche für Anatomie und für Physiologie war bereits in der Akademiezeit - sozusagen durch die Macht des Faktischen - erzwungen worden, als es galt, den 1819 nach Bonn berufenen Anatomen Carl Mayer zu ersetzen ${ }^{4}$. Für dessen Nachfolge meldeten sich Mayers Prosektor J. J. Hermann, der Stadtberner Dr. Ith und aus Stuttgart Dr. Rapp. Mit dem Argument, daß deutsche Gelehrte jeweils nach kurzen Gastspielen doch wieder in ihre angestammte Heimat abwandern würden, wurde Rapp nicht berücksichtigt. Bei den Probelehrveranstaltungen zeigte es sich, daß Hermann wohl über beachtliches anatomisch-präparatives Geschick verfügte, in der Physiologievorlesung aber nicht zu überzeugen vermochte, während es sich bei Iths Qualitäten gerade spiegelbildlich verhielt. Nach längern fakultären Diskussionen entschloß man sich, auf Probe Ith als Ordinarius die Physiologie und Hermann als Prosektor die Anatomie lesen zu lassen. Nach dieser niemanden befriedigenden Zwischenlösung entschied die Kuratel, das Führungsorgan der Akademie, im Frühjahr $1825^{1}$ die Anatomie definitiv durch A. Meckel aus Halle und die Physiologie durch Dr. Ith zu besetzen, aber keine eigenständigen Institute zu gründen ${ }^{4}$. Dieser Umstand, daß auch nach der Universitätsgründung die beiden getrennten Fächer bis 1894 unter dem gleichen, bald zu engen Dach vereint blieben,war für deren gedeihliche Weiterentwicklung wenig förderlich. 
In Zusammenhang mit der bevorstehenden Universitätsgründung hatte die Stadt 1832 vorsorglich den Vertrag für das alte, von der Akademie gemietete Anatomiegebäude, das im Garten des Mädchen-Waisenhauses stand und Ursache vielfältiger Unannehmlichkeiten war, gekündigt und so die neue liberale Regierung zur Errichtung eines Anatomie-Neubaus gezwungen. Bereits im Vorsommer 1832 wurden vom Bau-Departement Abklärungen über Raumbedarf und Standort in die Wege geleitet. Dabei galt es nicht nur die möglichen Bedürfnisse einer Anstalt für Anatomie und Physiologie im Rahmen einer eventuell zu gründenden eidgenössischen Universität abzuklären, sondern auch die Raumbedürfnisse der VeterinärAnatomie mit einzuplanen ${ }^{4}$.

Wenn man Hugo Kroneckers Ausführungen aus dem Jahre 1896 Glauben schenken will, bestand das im Herbst 1836 bezogene Physiologische Institut "als eines der ersten in deutschen Landen (sic) ... aus einem zum Hörsaal bestimmten zweifenstrigen Zimmer, einem schmalen Direktionszimmer und zwei einfenstrigen Arbeitszimmern im Erdgeschoß (des AnatomieGebäudes) und einem einfenstrigen Raum im 1. Stock, also 5 Zimmern»? ${ }^{7}$. Der nicht mehr existierende, klassizistische, symmetrisch gegliederte Axialbau (an der heutigen Genfergasse) wies auf der Gebäuderückseite neben einer eigentümlichen Abweichung von der Längsachsensymmetrie einen eigenartigen schräggestellten Halbrund-Anbau auf. Diese baulichen Absonderlichkeiten finden dadurch eine Erklärung, daß die Gebäuderückseite aus Teilen der alten Stadtringmauer unter Einbezug eines Wehrturms gefertigt war. Nach dem Bauwerkverzeichnis auf dem Abschnitt der Obern Stadt, Blatt II + III des Sickingerplans der Stadt Bern, muß es sich beim einbezogenen Wehrturm um den Lunthenturm gehandelt haben.

Gleich nach seiner Ankunft in Bern, am 10. Oktober 1836, ging Valentin daran, sein aus 5 Zimmern im neuen Anatomie-Gebäude bestehendes Institut mit studentischen Arbeitsplätzen für das Studium der Physiologie einzurichten, wie er dies bei seinem Lehrer Purkinje in Breslau kennengelernt hatte.

Die materiellen Forderungen, die Valentin bei seiner Berufung beim Erziehungs-Departement geltend machte, fielen - vergleicht man sie mit heutigen Usanzen - äußerst bescheiden aus. Sie betrafen ein Schreibpult mit Zubehör, einige Stühle und Tische zur Einrichtung von Arbeitsplätzen, einen abschließbaren Präparateschrank, einen wenn möglich eisernen Trocknungsofen mit großer Abstellfläche zur Herstellung von histologischen Präparaten sowie einen jährlich wiederkehrenden Apparatekredit von 
Fr. 100.-. Letzterer wurde im Studienjahr 1836/37 zur Anschaffung diverser Registrierapparaturen sowie für den Kauf von Glaswaren und Chemikalien verwendet ${ }^{5}$. Dazu konnte, dank den Bemühungen Valentins, unter zweckmässiger Verwendung eines ihm bewilligten Kredits von Fr. 500.-, die erforderliche Vervollständigung des «Apparates für vergleichende Anatomie» verwirklicht werden ${ }^{2}$.

Wenn nach Freiburg i.Br. 1821, Breslau 1824 jetzt auch Bern über ein selbständiges Physiologisches Institut verfügte, erhellt die Schilderung der apparativen Installationen des Berner Instituts schlaglichtartig - Frankreich ausgenommen - den Entwicklungsstand der europäischen Physiologie im ersten Drittel des 19. Jahrhunderts. Die immer noch stark morphologisch geprägten Forschungsansätze werden auch in Valentins Formulierungen in der Einleitung Seite 2 seines Lehrbuchs der Physiologie des Menschen offengelegt: "Die Anatomie dient der Physiologie in doppelt Beziehung. Zuvörderst liefert sie gewissermaßen, wenn ich mich so ausdrücken darf, die Buchstaben, in welcher die Sprache der physiologischen Lehre mitgeteilt werden muß. Denn jede genügende Erörterung des Ganges irgend einer Maschine setzt natürlicher Weise eine vollständige Kenntnis der Form, der Beschaffenheit und der Gliederung des Räderwerks voraus. ${ }^{18}$

Die nach Albrecht von Haller aufkeimende experimentelle physiologische Arbeitsrichtung eines Aloysius Luigi Galvani, Antoine Laurent Lavoisier oder Pierre Simon Laplace, geriet im deutschen Kulturraum rasch in den Sog des nachrevolutionären restaurativen romantischen Vitalismus der zehner und zwanziger Jahre des 19. Jahrhunderts. Nach den Vorstellungen des Modephilosophen Schelling war "Die Natur sichbarer Geist, der Geist dagegen unsichtbare Natur; der Organismus die Natur im Kleinen».11 Man konnte nach diesen Vorstellungen nur dadurch in das Innere der Natur eindringen, daß man hinter der Fassade der äußeren Erscheinungen ihre Ideen erkannte. Anstatt Kreislaufprobleme experimentell anzugehen, begannen die Physiologen, sich mit der Idee des Kreislaufs zu befassen. Fragen zum Einzelnen wurden als unergiebig, der ganzheitliche Ansatz - weg vom partikularen Experiment - für relevant gehalten. Johannes Müller, sicher einer der unverdächtigen Väter der modernen Physiologie, war zeitlebens nie ganz davon zu überzeugen, daß in lebenden Systemen neben den physikalisch-chemischen Gesetzen vitalistischen Kräften keine Bedeutung zukommen würde.

Die französische nachrevolutionäre Physiologie, vertreten durch Julien Jean César Legatois, Marie Jean Pierre Flourens und François Magendie, 
wurde von der romantisch-naturphilosophischen Modeströmumg kaum erfaßt. Diese französischen experimentell tätigen «vivisektorischen» Physiologen übten zusammen mit den deutschen Experimentalisten Ernst Heinrich Weber, Alfred Wilhelm Volkmann und anfänglich auch Jan Evangelista Purkinje Brückenfunktion für die Weiterentwicklung der experimentellen physikalischen Physiologie der sechziger Jahre.

Als Valentins intensive Zusammenarbeit mit Purkinje in Breslau begann, galten Purkinjes Hauptbemühungen - er hatte 1832 ein Mikroskop erhalten $^{3}$ - kaum mehr der experimentellen Sinnesphysiologie (PurkinjeBildchen, Purkinje-Phänomen usw.) als vielmehr der Histologie, Embryologie, der pflanzlichen und der tierischen Zytologie (Purkinje-Zellen des Herzens und des Kleinhirns), Forschungsrichtungen, denen auch Valentin schwergewichtig ein Leben lang verpflichtet blieb. Beide gehörten der Zwischengeneration von Gelehrten an, die bis über die Jahrhundertmitte hinaus vorab vergleichende Anatomie, Entwicklungsbiologie und eine vorwiegend beschreibende Physiologie in Lehre und Forschung in sich vereinigten.

Vor der Schilderung der 45 langen und bewegten Jahre in Bern soll an dieser Stelle Valentins Biographie kurz Erwähnung finden. Gabriel Gustav Valentin wurde am 8. Juli 1810 in der damaligen preußischen Stadt Breslau (dem heutigen Wrozlaw) als einziges Kind des Goldschmieds und Silberhändlers Aaron Valentin und der Carolina Bloch geboren ${ }^{6}$. Seine Mutter starb zwei Jahre nach Valentins Geburt ${ }^{14}$. In einem kleinbürgerlichen orthodox-jüdischen Milieu aufwachsend, erhielt er eine solide gymnasiale Ausbildung. Er las, sprachbegabt und interessiert, neben lateinischen und griechischen auch hebräische Texte und nahm am religiösen Leben aktiv Anteil. Trotz aller spätern beruflicher und gesellschaftlicher Nachteile, die ihm die Glaubenszugehörigkeit einbrachte, wich er zeitlebens nie von seinem angestammten jüdischen Glauben ab. Auf einer Silberstiftzeichnung von Johann Friedr. Dietler von 1842 (im Besitz des Medizinhistorischen Instituts der Universität Bern) fällt in den Gesichtszügen des jetzt 32 jährigen Valentin neben den kritisch sensitiven, melancholisch-mißtrauisch blickenden Augen und - fast im Gegensatz dazu - die Ausformung der Wangen-, Mund- und Kinnpartie auf, die jene Energie zum Festhalten, aber auch Rechthabenwollen und Rechtbekommenwollen durchscheinen lassen, die seinen ganzen Lebensweg prägten. Nach dem Hinschied des Vaters im November 1830 - Valentin studierte im zweiten Jahr Medizin - wurde ihm klar, daß er aus materiellen Gründen gezwungen sein würde, die Laufbahn 
eines praktizierenden Arztes einzuschlagen. Sofort nach dem Staatsexamen eröffnete er 1833 seine Praxis, die ihn aber nicht zu befriedigen vermochte. Dies war der Grund, neben seinem Brotberuf eine ihn mehr befriedigende Beschäftigung zu suchen. Wenngleich nach den biographischen Aufzeichnungen von Rudolf Heidenhain Purkinje vergleichsweise trockene Vorlesungen hielt, müssen Valentin aber schon als Student dessen außergewöhnliche wissenschaftliche Qualitäten nicht entgangen sein $^{3}$. Die Anstrengungen, mit seinem früheren Lehrer zusammenarbeiten zu können, waren erfolgreich. Anläßlich der Breslauer Jahresversammlung der deutschen Naturforscher und Ärzte von 1833 hielt Valentin ein vielbeachtetes Referat über eine Doppelmißbildung von Hühnerembryonen. Hier machte er die persönliche Bekanntschaft mit Johannes Müller und Alexander von Humboldt. Letzterer scheint seine Sympathie für Purkinje auch auf dessen Schüler übertragen zu haben und war sicher nicht unwesentlich daran beteiligt, daß Valentin 1835 den «Grand Prix des Sciences Physiques» in Paris zugesprochen erhielt. Das wichtigste Resultat der fruchtbaren Zusammenarbeit mit Purkinje war die Entdeckung der Flimmerepithelien, über das beide gemeinsam im Müller's Archiv 1834 berichteten ${ }^{9}$. Die kurz darnach abrupt einsetzende Entfremdung dürfte ihren Grund mehr in Valentins Verstimmung wegen nicht anerkannten Prioritätsansprüchen bei der Flimmerepithelentdeckung als in Streitereien um Bücher und um die Benützung des einzigen, im Labor von Purkinjes Privatwohnung stehenden Mikroskops gehabt haben, wie ein Brief Valentins an Purkinje vom 18. Juni 1855 das weismachen möchte ${ }^{20}$.

Der Verlust seines Arbeitsplatzes bei Purkinje wurde durch die Riesenarbeit an der mehr als 1000 Seiten umfassenden Preisschrift zum Thema «Examiner si la mode de développement des tissues organiques chez les animaux peut être comparé à la manière dont se fait le développement des tissues végétaux», das die französische Akademie der Wissenschaft ausgeschrieben hatte, überbrückt ${ }^{15}$. Parallel dazu verfaßte er 1835 sein «Handbuch der Entwicklungsgeschichte des Menschen mit vergleichender Rücksicht der Entwicklung der Säugetiere und Vögel» und zahlreiche weitere Kurzmitteilungen über verschiedene vorwiegend anatomische Beobachtungen ${ }^{16}$. Durch die Zusprache des Akademie-Preises von 3000 francs d'or, 1835, war Valentins Privatgelehrtendasein materiell vorerst gesichert.

Als anläßlich eines Berlin-Aufenthalts im März 1836 Johannes Müller und der Verleger Veit Valentin nahelegten, er sollte eine referierende Zeitschrift der Anatomie und Physiologie herausgeben, nahm er diese Gelegenheit sofort wahr ${ }^{5}$. Er, der mißtrauische, so leicht verletzliche Autor, 
verfügte jetzt von 1836 bis 1843 mit dem «Repertorium für Anatomie und Physiologie» über eine Art von wissenschaftlicher Privatzeitschrift und «Current Content», in der er jederzeit eigene Arbeiten unterbringen, laufend über den aktuellen Wissensstand in der Grundlagenmedizin berichten und Kollegen für Mitteilungen großzügig Gastrecht anbieten konnte ${ }^{17}$. Durch diese vor allem editoriale Tätigkeit, die seine beeindruckende wissenschaftliche Universalität noch unterstrich, war Valentin so bekannt geworden, daß er 1835 von der Universität Dorpat einen ersten Ruf als Lehrstuhlinhaber für Anatomie und Physiologie erhielt. Auch Lüttich und Basel hatten sich für den vielversprechenden jungen Physiologen aus Breslau interessiert. Valentins kategorische Weigerung, sich taufen zu lassen, führte in allen drei Fällen zum Abbruch der Verhandlungen. Günstig dagegen verliefen die Kontakte mit der Berner Fakultät und Regierung, die beide auf eine liberale Gesinnung festgenagelt, in der Religionszugehörigkeit kein Problem sahen, beziehungsweise offiziell nicht sehen durften. Valentin war der erste nicht getaufte jüdische Ordinarius im deutschen Sprachraum geworden.

Wie wir schon sahen, hat Valentin im Wintersemester 1836/37 seine Lehr- und Forschungstätigkeit in Bern begonnen. In der Populärphysiologie bezeichneten 3 Wochenstunden umfassenden Vorlesung sollte die Physiologie als Teil jeder Allgemeinbildung dargestellt werden. Dazu kamen 4. Wochenstunden für allgemeine Anatomie mit Demonstrationen und 2 Wochenstunden Entwicklungsgeschichte. Im Sommersemester waren ein 4stündiges Kolleg über experimentelle Pathologie, 4 Wochenstunden vergleichende Anatomie und eine Wochenstunde Vorlesung über Bildungshemmungen eingeplant ${ }^{5}$. Vergleicht man die Texte seines zweibändigen "Lehrbuchs der Physiologie des Menschen» (1.Auflage 1844) ${ }^{18}$ und des "Grundrisses der Physiologie von 1846» ${ }^{19} \mathrm{mit}$ den handgeschriebenen Vorlesungsnotizen von J. Uhlmann ${ }^{13}$, vermag man sich ein gutes Bild über Valentins breitangelegten, stark im Morphologischen verhafteten Physiologie-Unterricht zu machen.

Auffallend ist, daß Valentin, trotzdem er über die Entwicklung der französischen Physiologie durch eigene Anschauung gut informiert war, nur in kurzen episodischen Ansätzen tierexperimentell arbeitete. Mag sein, daß ihn schlechte Erfahrungen wie die Begebenheit, die Carl Vogt, einer seiner Schüler in Bern in seinen "Lebenserinnerungen» festgehalten hat, abhielten $^{21}$. Darnach war Valentin eben daran, an einem Metzgerhund die Funktionen der Zungennerven zu studieren, als durch Unachtsamkeit des Anatomiedieners das Tier mit klaffender Halswunde entlief, was zur 
Zusammenrottung einer aufgebrachten Metzgerzunft führte, die nur mit Mühe beruhigt werden konnte. Sicher spielte Valentins eher kritische Haltung dem Tierexperiment gegenüber ebenfalls mit hinein, wenn Moritz Schiff 1856 als Extraordinarius nach Bern berufen, hier seine in Frankfurt begonnenenUntersuchungen über den tierischen Zuckerstoffwechsel nicht fortführte; Schiff, der später durch seine extensive tierexperimentelle Tätigkeit in Florenz und Genf einer der Hauptverursacher für die verschärfte Gangart der britischen Antivivisektionsbewegung wurde ${ }^{12}$.

Das bringt uns in die Zeit, da Valentin, wie es Richard Feller in seiner Universitätsgeschichte formulierte, «in der Anatomie unumschränkt herrschte». ${ }^{1} 1853$ hatte der Anatonom Theile Bern verlassen, weil er nach einem Zitat in den Lebenserinnerungen des Zoologen Maximilian Perty «die Plackereien eines gewissen Kollegen nicht länger ertragen wollte». ${ }^{8}$ Die Vermutung, wer dieser Kollege hätte sein können, wird praktisch zur Gewißheit, wenn man an die sehr engen räumlichen Verhältnisse im Doppelinstitut, das zudem die Veterinär-Anatomie mit beherbergte, denkt, und wenn man die relativ ähnlichen Forschungsinteressen berücksichtigt sowie die Geschichte, daß der Extraordinarius Theile zum Leidwesen Valentins bei der Wahl zum Rektor 1842/43 dem Ordinarius der Physiologie vorgezogen worden war ${ }^{1}$.

In der Periode von 1853 bis 1863, da Valentin das Rad der Geschichte zurückdrehend in Personalunion die Fächer Anatomie und Physiologie wieder zusammenzuketten trachtete, war die Entwicklung der modernen Physiologie, sozusagen unbemerkt, an Bern vorbeigegangen. Die EnergieVerausgabung Valentins, die sich aus der Einmann-Direktion zweier Institute ergab, führte unausweichlich zu Mißstimmigkeiten auch mit der Fakultät und dem Erziehungsdepartement. Dazu kamen das eheliche Zerwürfnis, das Valentin zwang, seine beiden Kinder außer Landes erziehen zu lassen, und in der Folge die schwere Erkrankung und der Tod seiner Gattin. Sein einst beliebtes Lehrbuch war durch moderne Werke wie dasjenige von Carl Ludwig längst überflügelt, und Streitereien über divergierende Ansichten hatten viele frühern Kollegen entfremdet und ihn verbittern lassen.

Schließlich setzte 1863 der energische Erziehungsdirektor Kummer die ordentliche Besetzung des zehn Jahre verwaisten Anatomielehrstuhls durch Christoph Theodor Aebi aus Basel durch ${ }^{1}$. Valentin mußte von einem seiner wenigen treuen Freunde, Henle in Zürich, lange davon überzeugt werden, daß an einen Wegzug von Bern auf einen deutschen Lehrstuhl realistisch nicht mehr zu denken sei ${ }^{5}$. 
Trotz "Schiffbrüchen und Schicksal», wie Valentin sein Leben sah, verblieb er bis 1881, als er einen Schlaganfall erlitt, auf seinem Posten als Lehrstuhlinhaber, volle 90 Semester Physiologie unterrichtend! Eine nicht genau datierte Photographie aus dem 7.Lebensjahrzehnt (im Besitze des Medizinhistorischen Instituts der Universität Bern) zeigt das einprägsame Gelehrtengesicht mit der hohen wohlgeformten Stirne. Die ungebrochen kritisch-energischen Züge werden jetzt aber durch die Behäbigkeit der Leibesfülle und durch ein feines um Augen und Mundwinkel spielendes Lächeln gemildert. Valentin scheint sich im letzten Lebensjahrzehnt sukzessive in die Rolle des geachteten Nestors einer vergangenen stark morphologisch geprägten Physiologie am Übergang zur Moderne eingelebt zu haben. Die Fakultät gedachte in Dankbarkeit des Umstandes, daß Valentin einst in den dreißiger Jahren der Berner Hochschule Glanz und Statur verliehen hatte, als sie ihn anläßlich des 40 jährigen Professorenjubiläums und mit der Erneuerung des 50jährigen Doktortitels ehrte.

Aber es waren 1881 - 1884 Paul Grützner ${ }^{10}$, ein Heidenhain-Schüler, und ab 1885 Hugo Kronecker ${ }^{10}$, welche das Berner Physiologie-Institut ins 20. Jahrhundert führten.

\section{Literaturhinweise}

1 Feller, R.: Die Universität Bern 1834-1934. Bern 1935

2 Haag, F.: Die Sturm- und Drang-Periode der Berner Hochschule 1834-1854. Bern 1914

3 Heidenhain, R.: Johannes Evangelista Purkinje. In Allgem. Dtsch. Biogr. 26 (1888) 717-731

4 Hintzsche, E.: Die geschichtliche Entwicklung Anatomischer Arbeit in Bern bis zur Gründung der Hochschule. Bern 1942

5 Hintzsche, E.: Gabriel Gustav Valentin (1810-1883). Versuch einer Bio- und Bibliographie. Bern 1953

6 Kisch, B.: Gabriel Gustav Valentin (1810-1883). The Life of a Jewish Pioneer of Modern Medicine. In: Victor Robinson Memorial Vol.: Essays on History of Medicine. New York 1948

7 Kronecker, H.: Physiologisches Institut. In: Die Naturwissenschaftlichen und Medicinischen Institute der Universität Bern. Biel 1896

8 Perty, M.: Erinnerungen aus dem Leben eines Natur- und Seelenforschers des 19. J.H. Leipzig/Heidelberg 1876

9 Purkinje, J.E., Valentin, G.: Entdeckung continuierlicher durch Wimperhaare erzeugter Flimmerbewegungen als eines Phaenomens in den Klassen der Amphibien, Vögel und Säugetiere. In: Müller's Arch. f. Anat., Physiol. u. wiss. Med. 1 (1834) 391-400

Rothschuh, K.E.: Geschichte der Physiologie. Berlin 1953 
11 Schelling, F.W.J.: Aphorismen über Naturphilosophie und vorläufige Bezeichnung der Standpunkte der Medizin nach Grundsätzen der Naturphilosophie. In: Marcus, F. und Schelling, F.W.J. Jahrbuch der Medizin als Wissenschaft. Tübingen 1805

12 Schiff, M. In: Dreifuss, J.J.: Débat sur la vivisection. Cahiers Fac. Med. Nr.11. Genève 1985

13 Uhlmann, J.: Handgeschriebene Vorlesungsnotizen zu den Physiologie-Vorlesungen. In: G. Valentin's Bibl. Med. Hist. Institut, Universität Bern

14 Valentin, G.: Historiae Evolutionis Systematis Muscularis Prolusio. Diss. zootom. Wratislaviae (Breslau) 1832

15 Valentin, G.: Histiogenia comparata. Nie publizierte Preisschrift in Arch.Acad. Sci. Paris

16 Valentin, G.: Handbuch der Entwicklungsgeschichte des Menschen mit vergleichender Rücksicht der Entwicklung der Säugetiere und Vögel. Berlin 1835

17 Valentin, G.: Repertorium für Anatomie und Physiologie. Kritische Darstellung fremder und Ergebnisse eigener Forschung. Vol. 1 (bis Vol. VII). Berlin 1836

18 Valentin, G.: Lehrbuch der Physiologie des Menschen. 2 Bde. Braunschweig 1844

19 Valentin, G.: Grundriß der Physiologie des Menschen. Braunschweig 1846

20 Valentin, G.: Nachlaß, Mss. Hist. Helv. XXVIII, 63-69, Burger-Bibl. Bern

21 Vogt, C.: Aus meinem Leben. Stuttgart 1896

\section{Summary}

Short biographical notes covering life and main scientific interests of Gabriel Gustav Valentin, the first Jewish director of a Physiological Institute at a German speaking University are given.

The curriculum vitae of this ambitious scientist dramatically reflects the value-jugementproblems between form (Anatomy) and function (Physiology), which played such a critical role in the development of the Physiological Sciences during the last century.

Prof. Dr. med. Paul Müller

Physiologisches Institut

Bühlplatz 5

CH-3012 Bern 